\title{
Medical Quiz: Single Best Answer (SBA)
}

\section{Ahmed JUa}

\section{Question No. 01}

A 35-year-old man complains of a three-month history of intermittent excruciating headaches. They are very variable and occur from once a month to three times a week. The headaches are associated with extreme anxiety and sweating. On examination, the patient's blood pressure is $152 / 95 \mathrm{mmHg}$ and during palpation of the abdomen the patient's skin flushes red. The most likely diagnosis is:
A. Cluster headache
B. Phaeochromocytoma
C. Subarachnoid hemorrhage
D. Migraine
E. Temporal arteritis

\section{Question No. 02}

A 67-year-old woman presents to you with extensive scalp hair loss which has been getting progressively worse over the last year. You also notice thinning of the eyebrows. The patient's medical history is significant of hypertension, hypercholesterolemia and left-sided pulmonary embolism one year ago. You assess the patient's medication list. Which one of the following drugs could be responsible for causing generalized alopecia?
A. Aspirin
B. Warfarin
C. Simvastatin
D. Ramipril
E. Bendroflumethiazide

\section{Question No. 03}

A 16-year-old boy presents with a low-grade fever which started 1 week ago. The patient also reports feeling fatigued and indicates pain in his joints. His parents mention that he has been visiting the toilet more often

\footnotetext{
Author Information

a. Dr. Jamal Uddin Ahmed, Associate Professor of Medicine, BIRDEM General Hospital
}

than usual. A urine dipstick shows trace proteins, while a blood test shows raised eosinophils. The most likely diagnosis is:
A. Acute tubulointerstitial nephritis
B. Renal failure
C. Diabetes mellitus
D. UTI
E. Reactive arthritis

\section{Question No. 04}

A 29-year-old woman complains of a 1-week history of weakness and malaise. She has recently become a vegetarian and eats mostly green vegetables and drinks lots of tea during the day. She is apyrexial and has a Creactive protein $(\mathrm{CRP})<5$. You suspect an abnormality of the patient's iron stores. What is the most appropriate investigation to determine iron store levels?
A. Bone marrow biopsy
B. Serum ferritin
C. Serum transferrin
D. Total iron binding capacity
E. Serum iron

\section{Question No. 05}

A 35-year-old woman complains of a sharp central chest pain that is acutely exacerbated each time she moves, breathes in or lies flat. The pain tends to stay in the center of the chest but occasionally moves towards her neck and shoulders. The pain is relieved by sitting forward. The patient does not drink alcohol, is not diabetic and does not smoke. A pericardial rub is heard on auscultation. The most appropriate diagnostic investigation is:
A. CT calcium score
B. ECG
C. Serum amylase
D. Chest x-ray
E. Echocardiography 